\title{
BIOMASS ESTIMATION OVER TROPICAL RAINFORESTS USING GNSS-R ON-BOARD THE CYGNSS MICROSATELLITES CONSTELLATION
}

\author{
Hugo Carreno-Luengo, Guido Luzi, and Michele Crosetto \\ Centre Tecnòlogic de Telecomunicacions de Catalunya (CTTC/CERCA), Castelldefels (Barcelona), Spain \\ Tel.+349364529 00, E-mail: hugo.carreno@cttc.cat
}

\begin{abstract}
A performance assessment of the Cyclone Global Navigation Satellite System (CyGNSS) mission for biomass studies is presented in this paper over Congo and Amazon tropical rainforests. The functional correlation between CyGNSS-derived trailing edge TE and Avitabile et. al. Above Ground Biomass (AGB) pan-tropical data is evaluated using ICESat/GLASderived Canopy Height $(\mathrm{CH})$, SMAP-derived Vegetation Optical Depth ( VOD) and Polarization Index ( PI ). The main objective is to investigate the sensitivity of CyGNSS to biomass over dense forest canopies. The potential advantage of CyGNSS as compared to classical monostatic radar missions could rely on the increasing signal attenuation by the upwelling vegetation cover that reduces the coherent scattering component $\sigma^{\text {coh,0 }}$. This term can only be collected in a bistatic radar geometry. Ignoring measurement noise, preliminary results show that the use of $\mathrm{TE}$ allows to estimate AGB up to $\sim 400$ ton/ha and $\sim 250$ ton/ha over Congo and Amazon rainforests at low elevation angles $\theta_{\mathrm{e}} \sim[20,40]^{\circ}$. The analysis using the SMAP-derived microwave vegetation parameters shows that: a) VOD performs well even over rainforests, and b) PI could depend on the $\mathrm{CH}$ levels.
\end{abstract}

Index Terms - GNSS-R, CyGNSS, tropical forests coherent scattering, vegetation optical depth, polarization index

\section{INTRODUCTION}

Global Navigation Satellite Systems Reflectometry (GNSS-R) is a sort of multi-static L-band passive radar that allows to collect Earthreflected radio-navigation signals within a wide swath. GNSS-R was first proposed in 1993 by the European Space Agency (ESA). The first feasibility study from space corresponds to an experiment performed at the Jet Propulsion Laboratory (JPL) using the Spaceborne Imaging Radar-C (SIR-C) on-board the Space Shuttle. This experiment pushed-forward the development of GNSS-R. The United Kingdom (U.K.) Disaster Monitoring System-1 and the U.K. Techdemosat-1 provided the first datasets to the community. In 2012, CyGNSS 8-microsatellites mission was selected by NASA as an Earth Venture Space System. More recently, a GNSS-R experiment was performed at JPL using the radar receiver on-board the Soil Moisture Active Passive (SMAP) satellite [1]. Different concepts have been also explored such as the GNSS rEflectometry, Radio Occultation, and Scatterometry experiment on-board the International Space Station (GEROS-ISS) and the ${ }^{3} \mathrm{Cat}-2$ 6U CubeSat mission using a small antenna $\sim 0.2 \mathrm{~m} \mathrm{[2]}$.

Quantifying the global carbon cycle is essential to understand many of the dramatic changes taking place in the Earth system, particularly those resulting from the burning of fossil fuel and landuse change. Forests absorb, store, and release large amount of carbon. Therefore, they are a key component of the carbon cycle. Current space-borne L-band radar saturates at $\sim 150$ ton/ha. The future ESAmission BIOMASS is aimed to overcome this limitation using Pband and a 12-m antenna. In this work, the performance of CyGNSS for biomass monitoring is evaluated for first time over tropical rainforests. The work is organized as follows. Section 2 describes the methodology. Section 3 presents the biomass reference data. Preliminary results are analyzed in Section 4. Finally, Section 5 provides first conclusions.

\section{MATERIALS AND METHODOLOGY}

\subsection{SELECTED CyGNSS SCIENTIFIC OBSERVABLE}

CyGNSS Level 2.1 Science Data Record [3] is selected for this study. Original data are first filtered out using an equivalent "CyGNSS overall quality flag" over land surfaces.

Reflected delay waveforms $\mathrm{WF}_{\mathrm{r} \text {,raw }}$ are obtained from the original DDMs $\left\langle\left|Y_{r}(\tau, f)\right|^{2}\right\rangle$ at zero Doppler frequency:

$$
\mathrm{WF}_{\mathrm{r}, \mathrm{raw}}=\left\langle\left|\mathrm{Y}_{\mathrm{r}}(\tau, \mathrm{f}=0)\right|^{2}\right\rangle \text {. }
$$

The delay bin resolution of the original 17-lag waveforms $\mathrm{WF}_{\mathrm{r}, \text { raw }}$ is $\sim 0.2552$ GPS C/A chips. An optimum re-sampling and interpolation of the resulting 1700-lag waveforms is performed using a spline method to increase the accuracy of the waveforms, before applying the algorithms to extract the observables. Several observables can be considered to evaluate the sensitivity of $\sigma^{\text {coh,0 }}$ and the volume scattering term to forests biomass. The TE is selected is this paper. TE is defined as the lag difference between the $70 \%$ power threshold of the high- resolution waveforms $\mathrm{WF}_{\mathrm{r}, \text { threshold }}$ and the corresponding to the maximum power of the waveforms $\mathrm{WF}_{\mathrm{r}, \mathrm{peak}}[1]$ :

$$
\mathrm{TE}=\tau \mathrm{WF}_{\mathrm{r}, \text { threshold }}-\tau{ }^{\mathrm{WF}_{\mathrm{r}, \text { peak }}} .
$$

Different power thresholds are tested. The $50 \%$ power threshold is found to cut-off some data. On the other hand, the $90 \%$ power threshold provides a lower dynamic range as compare to the $70 \%$. Finally, it is worth to point out that the incoherent scattering term $\sigma^{\text {incoh, } 0}$ is the main contribution to $\mathrm{WF}_{\mathrm{r} \text {,threshold }}$, while both the coherent $\sigma^{\text {coh,0 }}$ and the incoherent $\sigma^{\text {incoh,0 }}$ scattering terms 
contribute to the peak power of the waveforms $\mathrm{WF}_{\mathrm{r} \text {,peak }}$.

\subsection{MICROWAVE VEGETATION PARAMETERS}

SMAP Enhanced L3 Radiometer Global Daily 9-km Level L3 SPL3SMP_E Version 1.0 product is used to generate the auxiliary vegetation parameters used in the work to complement the analysis of the experimental results [4]: 1) Vegetation Optical Depth (VOD ). The retrieval is based on a priori NDVI information obtained from visible-near infrared reflectance data from the NPP/JPSS VIIRS instrument, and land cover type assumptions. It is used to retrieve the transmissivity of the vegetation as an estimation of the attenuation of the electromagnetic signal through the vegetation layer. VOD is a function of the observation frequency and the vegetation type. It can be linearly related to Vegetation Water Content (VWC). 2) Polarization Index (PI). It is defined as the difference between the microwave radiometry brightness temperatures at $\mathrm{V}-$ pol $\mathrm{T}_{\mathrm{BV}}$ and $\mathrm{H}-$ pol $\mathrm{T}_{\mathrm{BH}}$, normalized to their average value as follows:

$$
\mathrm{PI}=\frac{\mathrm{T}_{\mathrm{BV}}-\mathrm{T}_{\mathrm{BH}}}{\left(\mathrm{T}_{\mathrm{BV}}+\mathrm{T}_{\mathrm{BH}}\right) / 2} .
$$

PI normalizes the measurements of the brightness temperatures and it is independent of the physical temperature of the surface. Additionally, it has been reported that it is independent of the vegetation type [5].

\subsection{STRATEGY: A GNSS-R BASED APPROACH}

TE values are classified into different groups according to different ranges of the satellites' elevation angles $\theta_{\mathrm{e}}$, in steps of $\sim 10^{\circ}$ from $\theta_{\mathrm{e}} \sim 90^{\circ}$ to $\theta_{\mathrm{e}} \sim 20^{\circ}$. $\theta_{\mathrm{e}}$ is an important parameter that determines the ratio of the coherent $\left\langle\left|Y_{r, \text { coh }}(\tau, f)\right|^{2}\right\rangle$ to incoherent $\left\langle\left|Y_{r, \text { incoh }}(\tau, \mathrm{f})\right|^{2}\right\rangle \quad$ scattering components. The soil surface contribution to the incoherent scattering term $\sigma^{\text {incoh,0 }}$ is filtered out to evaluate only the effect of the volume scattering term. In so doing, only pixels over the surface with a Terrain Ruggedness Index (TRI) lower than 15 are considered [6]. This threshold was found empirically to limit the effect of the roughness on $\left\langle\left|Y_{r}(\tau, f)\right|^{2}\right\rangle$.

Quantifying and analyzing the relationship of TE with AGB and $\mathrm{CH}$ is the main focus of this work. In so doing, SMAP-derived VOD and PI parameterss are additionally used to further analyze the results. As such, a common grid is required because of the variety of products with different footprint-sizes and spatio-temporal sampling characteristics. A $0.1^{\circ} \times 0.1^{\circ}$ latitude/longitude grid is selected. All products are averaged using a moving window of $0.2^{\circ}$ in steps of $0.1^{\circ}$. The spatial resolution is $\sim 20 \mathrm{~km} \times 20 \mathrm{~km}$ at equatorial latitudes, which is wide enough to account for the across-track spreading $\sim 20$ $\mathrm{km}$ of the DDMs due to $\left\langle\left|\mathrm{Y}_{\mathrm{r}, \text { incoh }}(\tau, \mathrm{f})\right|^{2}\right\rangle$. The ultimate resolution at a cell center is $\sim 20 \mathrm{~km} \times 20 \mathrm{~km}$ because multiple satellites groundtracks with different azimuthal angles are considered. This grid also allows to account for $\left\langle\left|Y_{r, \text { coh }}(\tau, \mathrm{f})\right|^{2}\right\rangle$. This term is limited by the along-track resolution $\sim 7.6 \mathrm{~km}$, related with the orbital motion and the incoherent integration time $1 \mathrm{~s}$. Finally, it is worth to point out that this strategy provides a filtering of potential fluctuations of TE due to remaining (after quality flag-removal) noise sources such as
Attitude Determination and Control Subsystem (ADCS), geolocation of the nominal specular reflection point, and the use of 8 different DDMs sources (8 distributed microsatellites) in the moving averaging filter.

The selected temporal window is 6-months $(01 / 08 / 2018$ to $31 / 01 / 2019$ ). This temporal length is large enough to provide statistically significant results. The temporal length was selected as large as possible at the time of starting this work. This specific timeperiod was selected because the Automatic Gain Control (AGC) was disabled in July 2018, so as to improve the performance in the estimation of the reflectivity. This study is not focused on the analysis of potential temporal fluctuations of different geophysical parameters such as Soil Moisture Content (SMC). The temporal variations of $\mathrm{AGB}$ and $\mathrm{CH}$ during this period are assumed to be negligible.

\section{REFERENCE DATA}

\subsection{ICESat/GLAS CANOPY HEIGHT MAP}

The selected reference canopy height data is the global map generated by Healey et al. using data collected by the Geoscience Laser Altimeter System (GLAS) lidar-instrument on-board the NASA's Ice, Cloud, and land Elevation Satellite (ICESat) from 2004 to 2008 [7]. GLAS is a waveform sampling lidar that uses three lasers, each one with a nominal lifetime of 18 months, and a spatial resolution of $\sim 60 \mathrm{~m}$ along-track and $\sim 170 \mathrm{~m}$ across- track. Only one laser operates at a time to achieve the nominal mission lifetime. GLAS transmits $\sim 40 \mathrm{~Hz}$ pulses using infrared light $\lambda \sim 1064 \mathrm{~nm}$ to detect changes on Earth's surface elevation and visible green light $\lambda \sim 532 \mathrm{~nm}$ to measure clouds and aerosol heights. In so doing, ICESat points $\sim 0.3^{\circ}$ off-Nadir during nominal operations to mitigate the potential damage of the detector due to specular reflections of laser pulses from mirror-like surfaces (e.g., calm water). ICESat mission includes estimation of vegetation height as a secondary mission objective. The shape of the Earth-reflected waveforms depends on the vertical distribution of vegetation and surface properties. Level L1a waveforms are used to calculate the so-called Lorey's height, which is the crown-area-weighted height. The applied algorithm uses a height correction factor based on the trailing waveform edge extent to correct for topographic effects because of the non-homogeneous sampling properties of GLAS. The final product is delivered with a spatial resolution $\sim 0.2 \mathrm{~km}$.

\subsection{PAN-TROPICAL ABOVE GROUND BIOMASS MAP}

The selected reference AGB data is the integrated pan-tropical map developed by Avitabile et al. [8]. This map combines Baccini's and Saatchi's AGB datasets into a $\sim 1 \mathrm{~km}$ resolution product. In so doing, it uses an independent reference of field observations for validation, and highly resolution biomass maps locally-calibrated, harmonized, and upscaled to 14,477 1 km AGB estimates as inputs for the fusion algorithm. The data fusion approach applies a bias removal and a weighted linear averaging. Baccini's and Saatchi's patterns use ICESat/GLAS as primary data source, similar strategy to upscale lidar-data, and they assume continental allometric relationships. However, Baccini's dataset covers tropical latitudes $[-23.4,23.4]^{\circ}$ while Saatchi's dataset has a longer coverage. As such, the fusion model is first applied to the common area and then it is extended to the area where Saatchi's dataset is available. In this region, the fusion algorithm only removes the bias of the Saatchi's dataset using the values estimated over Baccini's coverage. The validation of Avitabile's map shows a lower RMSE (reduction down to $\sim 74 \%$ ) and bias (reduction down to $\sim 153 \%$ ) than both original datasets over all the continents. 
(a)

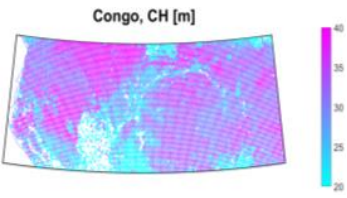

Amazon, $\mathrm{CH}[\mathrm{m}]$

(d)

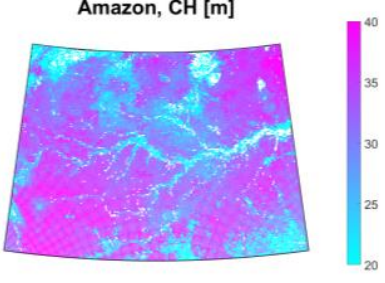

(b)

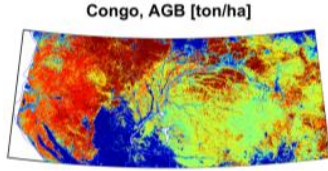

(e)

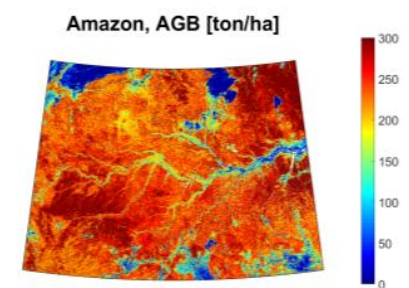

(c)

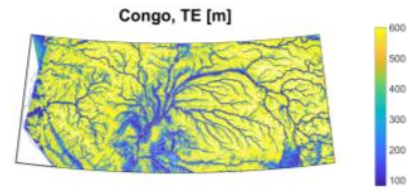

(f)

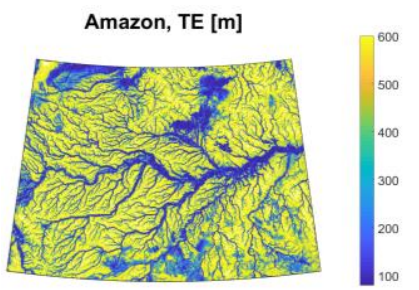

Fig. 1. Biomass study over Congo [(a) Canopy Height (CH), (b) Above Ground Biomass (AGB), (c) CyGNSS 70\% trailing edge ( TE ) width at $\theta_{\mathrm{e}}=$ $\left.[45,65]^{\circ}\right]$ and Amazon [(d) CH, (e) AGB, (f) CyGNSS 70\% trailing edge (TE) width at $\left.\theta_{\mathrm{e}}=[45,65]^{\circ}\right]$ tropical rainforests. Amazon: Lat. $=[-11,5]^{\circ}$, Lon. $=[-75,-54]^{\circ}$. Congo: Lat. $=[-4,4]^{\circ}$, Lon. $=[9,28]^{\circ}$.

\section{FIRST RESULTS}

The spreading of the TE is significant up high levels of biomass [Fig. 1]. AGB and CyGNSS sampling properties show a good performance, while GLAS-derived $\mathrm{CH}$ data are provided with less spatial density. This observation motivates the selection of AGB as the main biomass reference, while $\mathrm{CH}$ is used as an auxiliary product.

\subsection{SENSITIVITY AS A FUNCTION OF THE ELEVATION ANGLE}

Fig. 2 shows the scatter plots of TE and AGB over Congo [Figs. 2(a)-(d)] and Amazon [Figs. 2(e)-(h)] rainforests, as a function of $\mathrm{CH}$. They are computed using the mean values of TE in steps of $\sim 1 \mathrm{~m}$. This strategy allows to reduce noise at pixel level, such as speckle in $\mathrm{Y}_{r}(\tau, \mathrm{f})$ and potential errors in GLASderived AGB. The extension of the target areas is large, providing enough data. The errors are assumed to be randomly distributed. Thus, the noise can be reduced after averaging, so as to find the underlying functional correlation between TE and AGB.

TE increases as larger is the amount of AGB up to $~ 800 \mathrm{~m}$ over Congo [Fig. 2d] and $\sim 600 \mathrm{~m}$ over Amazon [Fig. (e)-(h)]. This point is explained because for increasing vegetation levels [Fig. 1]: (a) a higher volume scattering term in $\sigma^{\text {incoh, } 0}$ increases the tail of the waveforms, and (b) the coherent scattering term $\sigma^{\text {coh, } 0}$ is more attenuated. On the other hand, the AGB dynamic range increases with lower $\theta_{\mathrm{e}}$, from $\sim 140$ ton/ha at $\theta_{\mathrm{e}} \sim[80,90]^{\circ}$ to 300 ton/ha at $\theta_{\mathrm{e}} \sim[20,30]^{\circ}$ over the Congo. Over Amazon, it increases from $\sim 120$ ton/ha at $\theta_{\mathrm{e}} \sim[80,90]^{\circ}$ to 160 ton/ha at $\theta_{\mathrm{e}} \sim[20,30]^{\circ}$ over the Amazon. The improved dynamic range at lower angles could be explained because of the higher coherent reflectivity at this observation geometry. This characteristic increases the dynamic range of the Signal-to-Noise (SNR), which in turn belongs to a higher sensitivity to the upwelling attenuating cover.
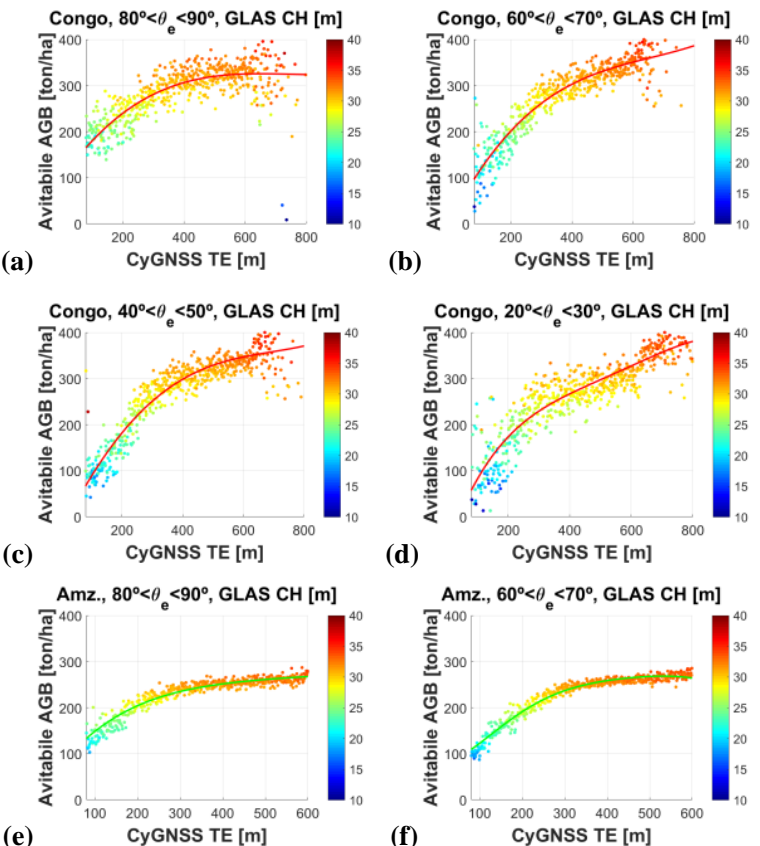

(d)

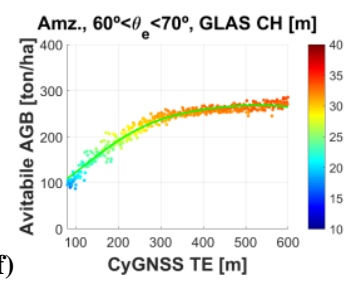

Amz., $40^{\circ}<\theta_{e}<50^{\circ}$, GLAS CH [m]
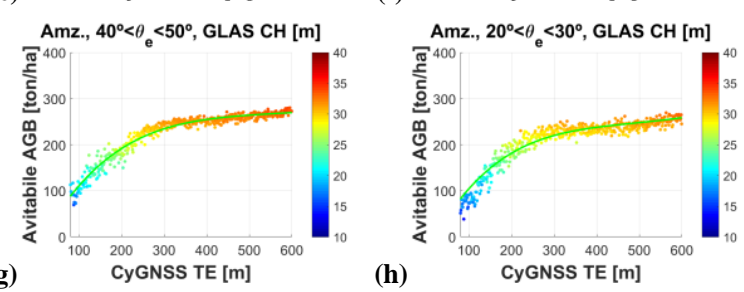

Fig. 2. Relationship between 6-months CyGNSS-derived trailing edge TE and Avitabile et al. AGB as a function of ICESat/GLAS CH over (a)(d) Congo and (e)-(h) Amazon rainforests as a function of the elevation angle $\theta_{\mathrm{e}}$.

Coherence effects could also appear after scattering over the vegetation cover if the coherent integration time would set to be long enough e.g. $20 \mathrm{~ms}$ [9], so as to filter out the noise and the volume scattering term. However, the volume scattering term is 


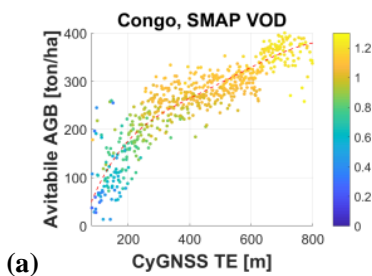

(a)

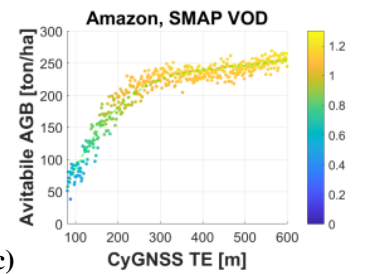

(b)
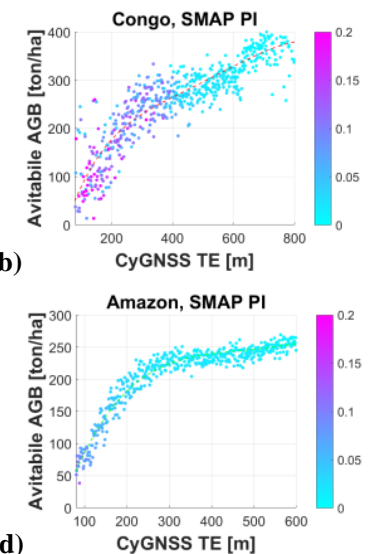

Fig. 3. Optimum relationship between 6-months CyGNSS-derived trailing edge TE with Avitabile et al. AGB over Congo and Amazon forests as a function of SMAP-derived VOD (a),(c), and PI (b),(d).

significant for lower integration times $\sim 1 \mathrm{~s}$.

The functional correlation of TE with AGB is fitted by empirically-derived polynomial functions at different angular ranges $\theta_{\mathrm{e}} \sim[80,90]^{\circ}, \sim[60,70]^{\circ}, \sim[40,50]^{\circ}$, and $\sim[20,30]^{\circ}$ over Congo and Amazon. The first derivative of these functions is computed at several AGB values [Table I]. The derivative values reduce for higher AGB levels. Ignoring noise in the measurements, this point is translated into smaller uncertainty in AGB estimation using the empirically-derived fitting functions. Assuming an error of e.g. $\sim 1 \mathrm{~m}$ in the estimation of $\mathrm{TE}$, preliminar results show that the uncertainty in AGB retrieval is as good as $\sim 0.3$ ton/ha for $\mathrm{AGB} \sim 350$ ton/ha at $\theta_{\mathrm{e}} \sim[20,30]^{\circ}$.

\subsection{EVALUATION AS A FUNCTION OF VOD AND PI}

At present, most of the biomass monitoring studies are based on visible and infrared indices. However, these types of sensors are only sensitive to the upper canopy layers, and they additionally suffer from weather conditions and clouds. This point motivates the study at-a-time of the performance of GNSS-R using vegetation measurable parameters i.e. AGB \& $\mathrm{CH}$ and microwave radiometryderived parameters such as VOD [10] \& PI [5]. The functional relationship between $\mathrm{AGB}$ and $\mathrm{TE}$ is evaluated only at the specific angular range where the optimum performance is found in terms of correlation coefficients and slope of the regressions [Fig. 3]. This is a case-by-case study, which depends on the characteristics of each type of forest. In case of Congo and Amazon, it is found that this optimum performance appears at $\theta_{\mathrm{e}} \sim[20,30]^{\circ}$. VOD increases as larger is the amount of AGB, without an apparent level of saturation. A significant sensitivity of VOD to AGB is found over Congo up to AGB $\sim 400$ ton/ha \& TE $\sim 800 \mathrm{~m}$ [Figs. 3(a),] and over Amazon up to AGB $\sim 250$ ton/ha \& TE $\sim 600 \mathrm{~m}$ [Figs. 3(c)]. As such, it can be stated that SMAP-derived VOD performs well even over rainforests. The sensitivity is rather similar to that of $\mathrm{CH}$ [Figs. 2(d),(h)]. On the other hand, PI decreases as higher is AGB because radiation from canopy is much less polarized than from soil. Polarized emissivity from homogeneous and smooth soils is attenuated by the volumetric effect of the upwelling vegetation cover. As such, this
Table I. First-derivative [(ton/ha)/m] polynomial fit of CyGNSS-derived TE over Congo and Amazon rainforests at $\theta_{\mathrm{e}} \sim[20,30]^{\circ}$.

\begin{tabular}{cccccccc}
\hline AGB [ton/ha] & $\mathbf{5 0}$ & $\mathbf{1 0 0}$ & $\mathbf{1 5 0}$ & $\mathbf{2 0 0}$ & $\mathbf{2 5 0}$ & $\mathbf{3 0 0}$ & $\mathbf{3 5 0}$ \\
\hline Congo & 1.53 & 1.21 & 0.90 & 0.55 & 0.35 & 0.33 & 0.30 \\
\hline Amazon & $\mathrm{x}$ & 1.07 & 0.75 & 0.40 & 0.10 & $\mathrm{x}$ & $\mathrm{x}$ \\
\hline
\end{tabular}

experimental observation suggests that PI could depend also on structural effects i.e. $\mathrm{CH}$ in addition of $\mathrm{VWC}$, at least over tropical rainforests.

\section{CONCLUSIONS}

Earth-reflected L1 GPS signals as collected in a bistatic radar configuration by CyGNSS using down-looking LHCP antennas with relatively low gain $\sim 15 \mathrm{~dB}$ show a significant sensitivity to $\mathrm{AGB}$ and $\mathrm{CH}$ over tropical rainforests without an apparent signal saturation level. First derivatives of the polynomial fitting functions of the relationship of AGB and TE are computed as a function of AGB. Based on the derivate values and ignoring measurement noise, preliminary results show that is possible to estimate AGB up to $\sim 400$ ton/ha and 250 ton/ha with errors $\sim 0.3$ ton/ha and $\sim 0.1$ ton/ha respectively over Congo and Amazon at $\theta_{\mathrm{e}} \sim[20,30]^{\mathrm{o}}$.

\section{REFERENCES}

[1] H. Carreno-Luengo, S. T. Lowe, C. Zuffada, S. Esterhuizen, and S. Oveisgharan, "Spaceborne GNSS-R from the SMAP mission: first assessment of polarimetric scatterometry over land and cryosphere," MDPI Remote Sensing, vol. 9. no. 361, pp. 362, Apr. 2017.

[2] H. Carreno-Luengo, A. Camps, P. Vila. J.F. Munoz, A. Cortiella, D. Vidal, J. Jané, N. Catarino, M. Hagenfeldt, P. Palomo, and S. Cornara, "3Cat-2; an Experimental Nano-Satellite for GNSS-R Earth Observation: Mission Concept and Analysis," IEEE JSTARS, vol. 9, no. 10, pp. 4540-4551, 2016.

[3] CYGNSS. 2017. CYGNSS Level 1 Science Data Record. Ver. 2.1. PO.DAAC, CA, USA. [Online]. Available http://dx.doi.org/10.5067/CYGNS-L1X20. (11/9/2019) [4] P. E. O'Neill, S. Chan, E. G. Njoku, T. Jackson, and R. Bindlish. 2016. SMAP Enhanced L3 Radiometer Global Daily 9 km EASE-Grid Soil Moisture, Version 1. [SPL3SMP_E]. Boulder, Colorado USA. NASA National Snow and Ice Data Center $\begin{array}{lll}\text { Distributed Active Archive Center. [Online]. Available } & \end{array}$ https://doi.org/10.5067/ZRO7EXJ8O3XI. (4/2/2019)

[5] E. Santi, S. Paloscia, P. Pampaloni, S. Pettinato, T. Nomaki, M. Seki, K. Sekiya, and T. Maeda, "Vegetation water content retrieval by means of multifrequency microwave acquisitions from AMSR2," IEEE JSTARS, vol. 10, no. 9, pp. 3861-3873, Sep. 2017.

[6] G. Amatulli, S. Domisch, M.-N. Tuanmu, B. Parmentire, A. Ranipeta, J. Malczyk, and W. Jetz, "A suite of global cross-scale topographic variables for environmental and biodiversity modelling," Nature Scientific data, Mar. 2018. DOI: 10.1038/sdata.2018.40 .

[7] S. P. Healey, M. W. Hernandez, D. P. Edwards, M. A. Lefsky, E. Freeman, P. L. Patterson, E. J. Lindquist, and A. J. Lister. 2015. CMS: GLAS LiDAR-derived Global Estimates of Forest Canopy Height, 2004-2008. ORNL DAAC, Oak Ridge, Tennessee, USA. [Online]. Available http://dx.doi.org/10.3334/ORNLDAAC/1271 (2/2/2019)

[8] V. Avitabile et al., "An integrated pan-tropical biomass map using multiple reference datasets," John Wiley and Sons Ltd., Global Change Biology, vol. 2, no.4, pp. 1406-1420. Apr. 2016. [Online]. Available https://doi.org/10.1111/gcb.13139 $(2 / 11 / 2018)$

[9] H. Carreno-Luengo, A. Camps, J. Querol, and G. Forte, "First results of a GNSS-R experiment from a stratospheric balloon over boreal forests," IEEE TGRS, vol. 54, no. 5, pp. 2652-2663, Dec. 2015.

[10] H. Carreno-Luengo, G. Luzi, and M. Crosetto, "Sensitivity of CyGNSS bistatic reflectivity and SMAP microwave radiometry brightness temperature to geophysical parameters over land surfaces,” IEEE JSTARS, 2018.

ACKNOWLEDGEMENTS This research was carried out with the support grant of a Juan de la Cierva research fellowship from the Spanish Ministerio de Economia, Industria y Competitividad (MINECO), reference FJCI-2016-29356. Travel expenses were partially covered by the Postdoctoral Award sponsored by the open access journal Remote Sensing published by MDPI. 